\title{
Modelling of new generation plasma optical devices
}

Irina V. Litovko,

Alexy A. Goncharov,

Andrew N. Dobrovolskiy,

Lily V. Naiko,

Irina V. Naiko

\begin{abstract}
The paper presents new generation plasma optical devices based on the electrostatic plasma lens configuration that opens a novel attractive possibility for effective high-tech practical applications. Original approaches to use of plasma accelerators with closed electron drift and open walls for the creation of a cost-effective low-maintenance plasma lens with positive space charge and possible application for low-cost, low-energy rocket engine are described. The preliminary experimental, theoretical and simulation results are presented. It is noted that the presented plasma devices are attractive for many different applications in the state-of-the-art vacuum-plasma processing.
\end{abstract}

Key words: anode layer $\bullet$ plasma lens $\bullet$ plasma accelerator $\bullet$ plasma jet $\bullet$ space charge

\section{V. Litovko}

Plasma Technology and Plasma Physics Department, Institute for Nuclear Research NASU,

47 Nauki pr., 03650 Kiev, Ukraine,

Tel.: +38 044525 2445, Fax: +38 044525 2329,

E-mail: ilitovko@rambler.ru, ilitovko@kinr.kiev.ua

\author{
A. A. Goncharov, A. N. Dobrovolskiy, L. V. Naiko, \\ I. V. Naiko \\ Gas Electronics Department, \\ Institute of Physics NASU, \\ 46 Nauki Ave., Kiev, 03028, Ukraine
}

Received: 20 September 2015

Accepted: 3 December 2015

\section{Introduction}

The crossed electric and magnetic fields, inherent to the cylindrical electrostatic plasma lens (PL) configuration, provide an attractive method for establishing a stable plasma discharge at a low pressure [1]. One particularly interesting result of this background work was the observation of the essential positive potential at the floating substrate. This suggested that an electrostatic PL can possibly be used for focusing and manipulating high-current beams of negatively charged particles (electrons and negative ions) that is based on the use of the dynamical cloud of positive space charge under the conditions of electron's magnetic insulation. An attractive possibility of the application of dynamical positive space charged plasma lens with a magnetic electron insulation and non-magnetised ions, for focusing and manipulating wide-aperture high-current negatively charged particles beams, has been shown in our previous works $[2,3]$. Here we describe the original approach to use plasma accelerators with a closed electron drift, which (unlike the traditional accelerators with metal and dielectric walls [4]), have open (gaseous) walls and can be applied for the creation of cost-effective low-maintenance plasma devices. They might be based on a plasma lens configuration for the production of accelerated ion beams converging towards the $z$-axis, and for the design of positive space charge plasma lens [5]. It has been shown that, based on the idea of the continuity of current transferring at the system in the frame of 
one-dimensional model, exact analytical solutions describing electric potential and electron density distribution along an acceleration gap can be found.

\section{Plasma accelerator with gas walls and closed electron drift}

\section{Set-up and preliminary experimental results}

Accelerators with closed electron drifts and open (gas) walls have not been studied till now, in contrast to the well-known and widely used plasma accelerators with an anode layer and accelerators with closed electron drifts and dielectric walls [4]. The accelerator with closed electron drifts can, however, be interesting for the optimisation of high current flows of charged particles and can be attractive for the elaboration of low-cost, effective and low maintenance plasma lens with a positive space charge cloud. The pilot experimental plasma accelerator with closed electron drifts and open walls is shown in Fig. 1a.

Such a cylindrical Hall-type plasma-ion source, which produced ion-plasma flows converging towards the symmetry axis of the system, was created for the exploration. The diameter of anode was $6.7 \mathrm{~cm}$, and that of cathode was $3.2 \mathrm{~cm}$. A distance between anode and cathode was equal to $1.75 \mathrm{~cm}$. Magnetic field

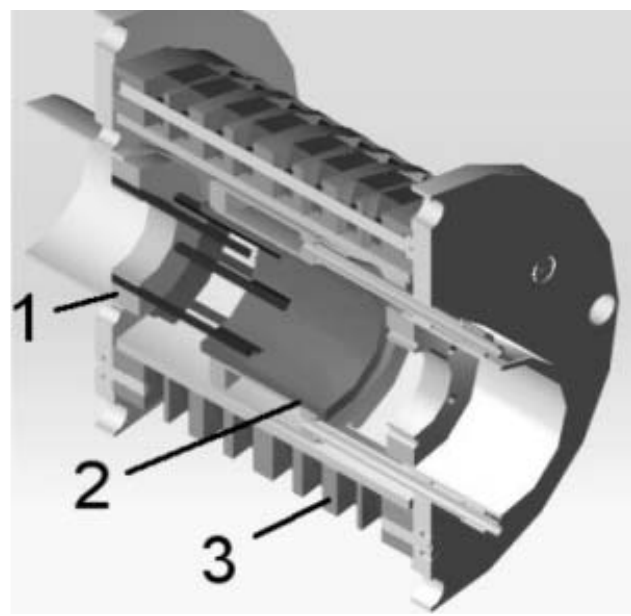

Fig. 1a. Experimental unit: 1 - cathode, 2 - anode, 3 permanent magnets system.

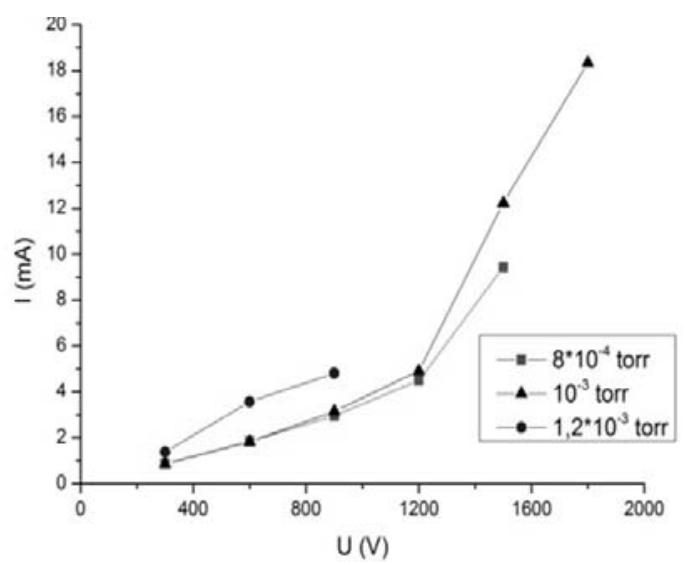

Fig. 1b. The total discharge current vs discharge potential for different work gas pressures.
$H=650-750$ Oe was created by permanent magnets. The applied voltage was below $2 \mathrm{kV}$. The working pressure was in a range of $10^{-5}$ to $10^{-3}$ Torr, and the working gas was pure argon. The formation sufficient amount of ions was possible due to the presence of magnetic field, which isolate anode from cathode.

Figure $1 \mathrm{~b}$ shows volt-ampere characteristics measured for different work gas pressure. Two operating modes were observed and the current-voltage characteristics of the accelerator in these modes were defined. We have low-current operation mode at a low pressure of the working gas, when all ionisation processes occur in an anode layer. At a pressure increase, the anode layer size grows up and the accelerator proceeds to a high-current mode with total current ranging up to $2 \mathrm{~A}$. In this mode, the discharge current is greater by several orders, but discharge is not localised near the anode any more, and can fill up the whole inner volume. In a high-current quasi-neutral plasma mode of the accelerator operation, a plasma jet is usually observed, as shown in Fig. 2a. These preliminary results show that potential drop appears along the jet axis and can be used for the ion beam acceleration.

Figure $2 \mathrm{~b}$ shows an ion beam current density dependence on current discharge along the jet axis. It should be noted that the power ion plasma flow increases with an increase in the discharge current density. One should also note that the ion current density at the jet axis can reach up to $2-3 \%$ of the total discharge current. That opens a novel attractive

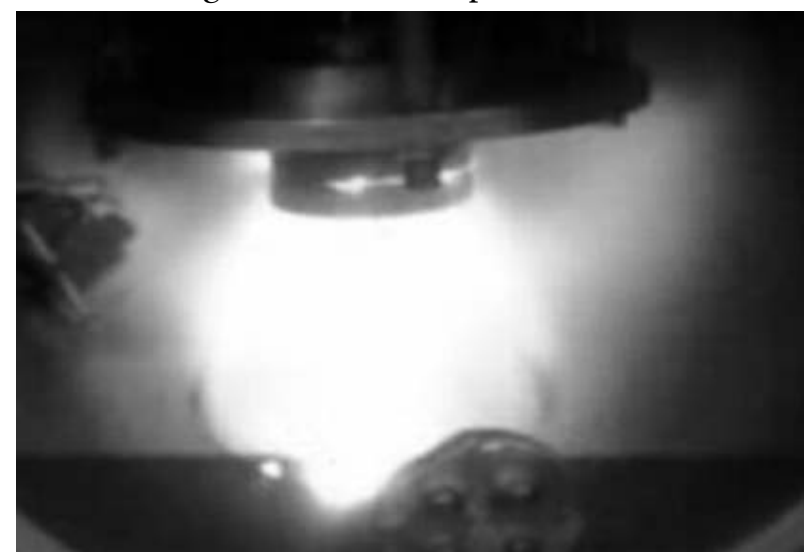

Fig. 2a. Plasma jet in a high-current operation mode. The plume is observed perpendicular to the acceleration direction.

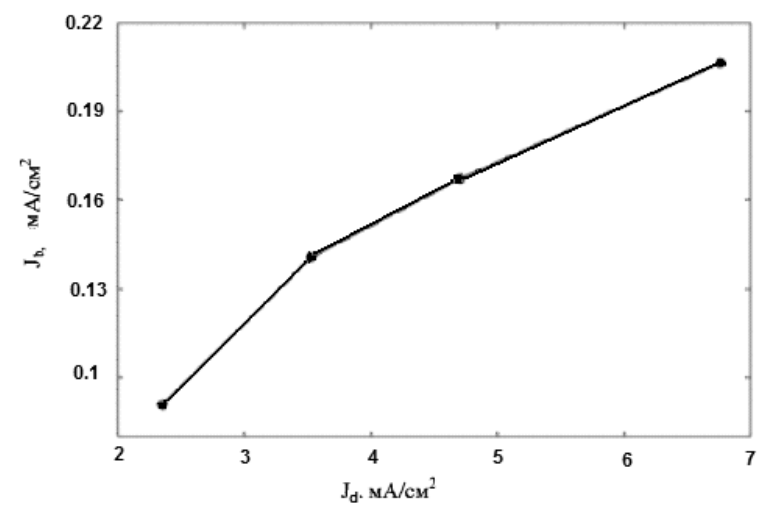

Fig. 2b. Dependence of a beam current density vs. discharge current density. 


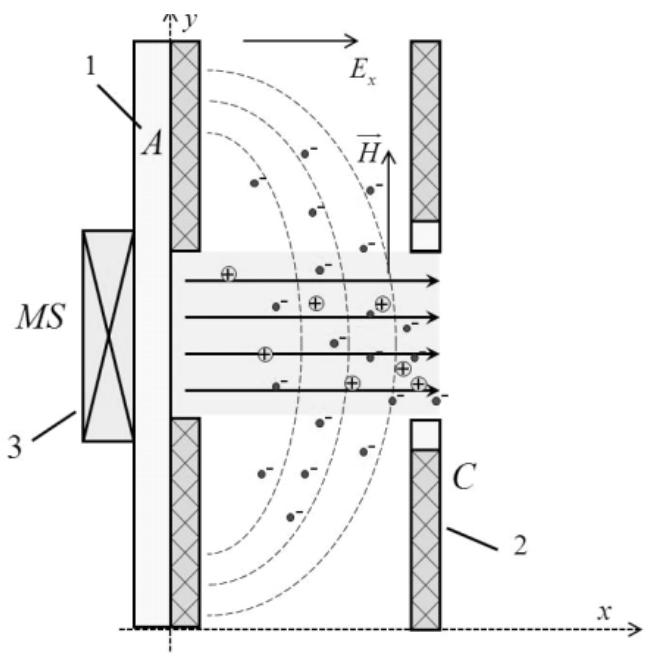

Fig. 3. Scheme for a theoretical model: 1 - anode, 2 cathode, 3 - magnetic system.

possibility to use this kind device as a prototype of small rocket engines.

\section{Model and analytical solutions}

In order to analyse the properties of the considered accelerator, we used a one-dimensional hydrodynamic model. A scheme used in this model is schematically shown in Fig. 3.

In the considered system, electrons are magnetised, ions are non-magnetised and move from the anode to the system axis. We can assume that a current density in the gap volume is the sum of the ion and electron components:

$$
j_{\mathrm{e}}+j_{i}=j_{d}
$$

where $j_{i}$ and $j_{\mathrm{e}}$ are ion and electron current density, respectively.

Consequently,

$$
\begin{aligned}
& j_{i}=e v_{i} \int_{0}^{x} n_{\mathrm{e}}(x) d x \\
& j_{\mathrm{e}}(x)=e \mu_{\perp}\left(n_{\mathrm{e}} E(x)-\left(n_{\mathrm{e}} T_{\mathrm{e}}\right)_{x}^{\prime}\right)
\end{aligned}
$$

where $v_{i}$ is the ionisation frequency, $\mu_{\perp}=e v_{i} / m \omega_{\mathrm{eH}}^{2}$ is the electron transverse mobility, $E(x)=-\phi_{x}^{\prime}$ is the electric field, $\phi$ is potential, $n_{\mathrm{e}}$ is the frequency of elastic collisions with neutrals and ions, $\omega_{\mathrm{eH}}$ is the electron cyclotron frequency, $T_{\mathrm{e}}$ is an electron temperature that can be found from the equation of the electrons heating in an electric field [4], that is,

$$
T_{\mathrm{e}}(x) j_{\mathrm{e}}(x)=\beta \int_{0}^{x} j_{\mathrm{e}}(s) \phi_{s}^{\prime} \mathrm{d} s
$$

where $0<\beta \leq 1$.

Thus, taking into account the values from Eqs. (1) and (2), we obtain the expression

$$
e v_{i} \int_{0}^{x} n_{\mathrm{e}}(x) \mathrm{d} x-e \mu\left(n_{\mathrm{e}} \phi_{x}^{\prime}+\left(n_{\mathrm{e}} T_{\mathrm{e}}\right)_{x}^{\prime}\right)=j_{d}
$$

Another equation for the analysed system description is the Poisson's equation:

$$
\phi_{x x}^{\prime \prime}=4 \pi e\left(n_{\mathrm{e}}-n_{i}\right)
$$

where for ion density we can write the expression

$$
n_{i}(x)=\sqrt{M / 2 e} \int_{0}^{x} \mathrm{~d} s n_{\mathrm{e}}(s) v_{i} / \sqrt{\phi(x)-\phi(s)}
$$

Considering the fact that ion current is equals on the cathode and taking into account a boundary condition $\left.\phi\right|_{x=0}=\phi$, where $\phi_{a}$ is an anode potential, we obtain the closed system of equations. In some cases, it is possible to find exact analytical solutions of this system. For example, neglecting a diffusion in the case when $n_{\mathrm{e}}>>n_{i}$, which corresponds to a low-current mode, we can get the solution in a dimensionless form:

$$
\phi=\left((x-1)^{2}-1\right) / 2 \alpha+1, \alpha=\mu_{\perp} \phi / v_{i} d^{2}
$$

where $d$ is the gap length. Potential distribution (Eq. (7)) for different values of parameter $\alpha$ is shown in Fig. 4 a.

One can see that at $\alpha=1 / 2$, the total applied potential falls inside the accelerating gap. In this optimal case, the gap length is:

$$
d=\sqrt{2 \mu_{\perp} \phi_{a} / v_{i}}
$$

Under assumption that all electrons originated

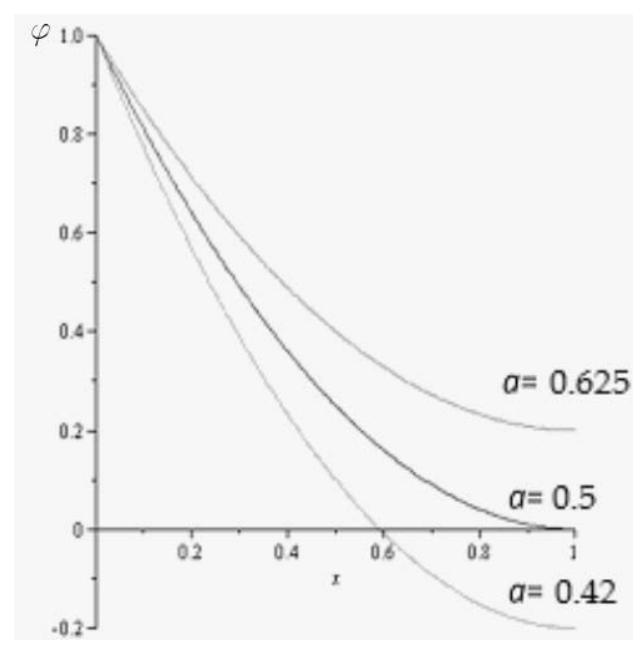

Fig. 4a. Potential distribution for different $\alpha$ values in a low-current mode.

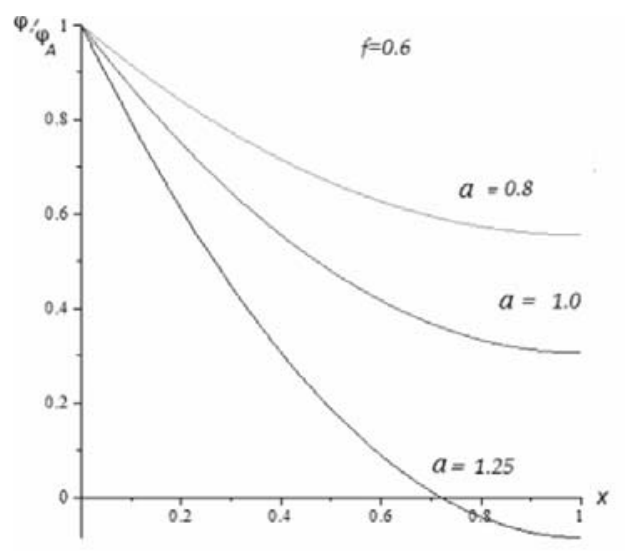

Fig. 4b. Potential distribution for different $\alpha$ values in a high-current quasi-neutral plasma mode. 
from the gap only by impact ionisation, and go to the anode due to classical transverse mobility, the previous expression can be represent in the form:

$$
\delta=\rho_{\mathrm{e}}\left(\phi_{a}\right) \sqrt{2 v_{\mathrm{e}} / v_{i}}
$$

This expression coincides with the one for classical anode layer [6] accurate within $\sqrt{ } 2$.

It should be noted that in the case when parameter $\alpha>1 / 2$ (the gap length is smaller than $\delta$ ), the potential drop is not completed. For the case $\alpha<1 / 2$, when the gap length $d>\delta$, the potential drop exceeds the applied potential. This can be due to an electron space charge dominating at the accelerator exit.

One can extend now our description taking into consideration $n_{i}$ and $n_{\mathrm{e}}$ changes along the gap, which corresponds to a high-current mode. As before, we will study the case $T_{\mathrm{e}}=0$ only. Then for dimensionless Eqs. (4), one can write the equation

$$
c \int_{0}^{x} n_{\mathrm{e}}(s) \mathrm{d} s-b n_{\mathrm{e}}(x) \phi_{x}^{\prime}=1
$$

where $b=\mu_{\perp} \phi_{a} e n_{0} / j_{d} d$ and $c=v_{i} d e n_{0} / j_{d}$ are dimensionless parameters.

For simplicity, we will consider quasi-neutral plasma $n_{\mathrm{e}} \approx n_{i}$. Then substituting a dimensionless equality (6) in Eq. (10), one gets

(11) $\int_{0}^{x} n_{\mathrm{e}}(s) \mathrm{d} s-b \cdot f \cdot \phi_{x}^{\prime} \int_{0}^{x} \mathrm{~d} s n_{\mathrm{e}}(s) / \sqrt{\phi(x)-\phi(s)}=1$

where $f=v_{i} d \sqrt{M / 2 e \phi_{a}}$. After some transformations and taking into account the fact that ion current equals $j_{d}$ upon the cathode, Eq. (11) could be rewritten in a form

$$
\int_{x}^{1} \mathrm{~d} s n_{\mathrm{e}}(s)(2 b f \sqrt{\phi(x)-\phi(s)})_{x}^{\prime}=\int_{x}^{1} \mathrm{~d} s n_{\mathrm{e}}(s) \cdot c
$$

Taking into account the equality of the considered integrals and boundary condition, one obtains the expression for the potential distribution:

$$
\phi(x)=1-a^{2} / 4 f^{2}+a^{2}(x-1)^{2} / 4 f^{2}
$$

where $a=c / b$. It should be noted that it is corresponding to the parameter $1 / \alpha$ introduced above. From Eq. (13), we can find that the behaviour of the potential distribution depends on a ratio $p=a^{2} / 4 f^{2}$. It should also be noted that the parameter $f$ describes the influence of the ion density (see Fig. 4b).

If we now derive Eq. (10), we could obtain an equation for the electron density:

$$
\left(n_{\mathrm{e}}(x)\right)^{\prime} / n_{\mathrm{e}}(x)=\left(a-\phi^{\prime \prime}\right) / \phi^{\prime}
$$

The solution of Eq. (14), at the given relation (13), has a form:

$$
n_{\mathrm{e}}(x)=\mathrm{C}(x-1)^{\frac{2 f^{2}}{a}-1}
$$

where $\mathrm{C}$ is a constant. One should note that if $a=$ $2 f^{2}$, the electron density does not change along the gap and the solution (13) is reduced to the value given by Eq. (7). The condition mentioned above could be rewritten in a form

$$
\tau_{e d} / \tau_{i d}^{2}=2 v_{i} \text { or } \tau_{e d} v_{i}=2 \tau_{i d}^{2} v_{i}^{2}=1
$$

where $\tau_{e d}=d / \mu_{\perp} E$ is electron lifetime, and $\tau_{i d}=$ $d / v_{i d}$ is ion living time. Indeed Eq. (16) is some generalisation condition of a self-sustained discharge in the crossed $E \times H$ fields, taking into consideration both electron and ion dynamics peculiarity.

\section{Results of simulations in hydrodynamic and hybrid models}

Now let us consider a more general description, assuming that electron heating losses occur mostly by different kinds of collisions. Introducing a characteristic time $\tau_{0}$ of energy losses, we can write a definition for the temperature:

$$
T_{\mathrm{e}}=j_{d} E \tau_{0}\left(1-e^{-t / \tau 0}\right) / e n_{\mathrm{e}}
$$

and for a steady-state

$$
T_{\mathrm{e}}=j_{d} E \tau_{0} / e n_{\mathrm{e}} m_{b b b b}
$$

Thus Eq. (4) can be rewritten in the form

$$
\mu_{\perp} \tau_{0} j_{d} \phi_{x x}^{\prime \prime}-e \mu_{\perp} n_{\mathrm{e}} \phi_{x}^{\prime}+e v_{i} d \int n_{\mathrm{e}} d x=j_{d}
$$

Making this equation and Poisson's equation dimensionless, that is, introducing dimensionless parameters, we can obtain a system of equations

$$
a \phi_{x x}^{\prime \prime}-b n(x) \phi_{x}^{\prime}+c \int_{0}^{x} n(s) \mathrm{d} s=1
$$

and

$$
n(x)-f \int_{0}^{x} n(s) \mathrm{d} s / \sqrt{\phi(x)-\phi(s)}=g \phi_{x x}^{\prime \prime}
$$

where the introduced new dimensionless parameters are $a=\mu_{\perp} \tau_{0} \phi_{a} / d^{2}$ and $g=\phi_{a} / 4 \pi d^{2} e n_{0}$, while parameters $b, c$ and $f$ correspond to those introduced above. In a general case, the considered equation system does not have analytical solutions and requires numerical calculations. If we assume a zero electric field on the cathode, we got solutions (see Fig. 5a) similar to obtained above (see Fig. 4a).

As one can see from Fig. 5b, the electron density changes by a small amount along the gap, thus our above consideration that $n_{\mathrm{e}}$ is constant along the gap is untrue. Note also that if we assume that $\tau_{0}=$ $\tau_{e d}$, then Eq. (17) will be reduced to the form: $T_{\mathrm{e}}=$ $j_{d} / \mu_{\perp} e n_{\mathrm{e}}$, consequently in Eq. (4), the temperature term disappears. Therefore, we come back to potential distribution (Eq. (7)).

For a more detailed study of the influence of ion dynamics on the system process, we applied a one-dimensional hybrid model used for calculations of a span mode with neutral-particle ionisation. In this model, ions and neutral dynamics is described by kinetic equations, where right parts take into account only a single ionisation: 


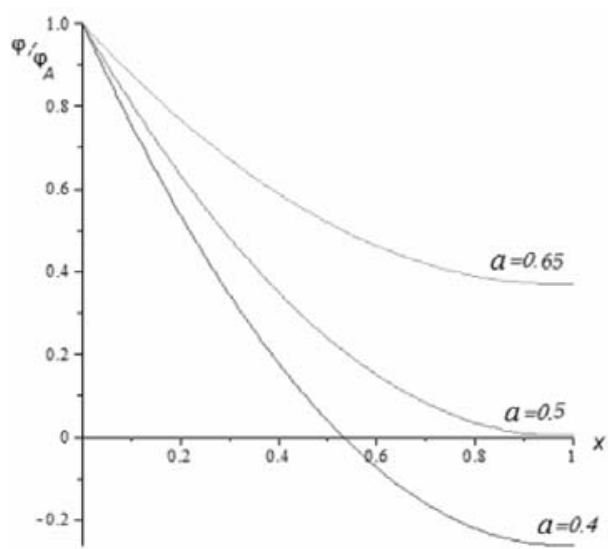

Fig. 5a. Potential distribution obtained from the numerical simulations.

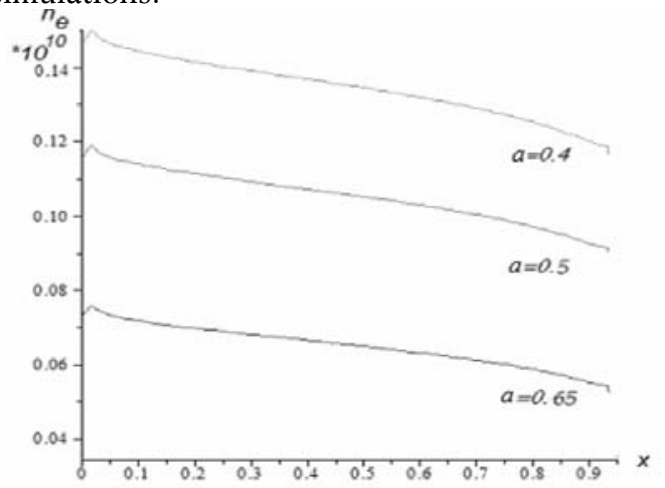

Fig. 5b. Electron density distribution obtained from the numerical simulations.

$$
\begin{aligned}
& \frac{\partial f_{0}}{\partial t}+v_{0} \frac{\partial f_{0}}{\partial x}=-\left\langle\sigma_{i e} v_{\mathrm{e}}\right\rangle n_{\mathrm{e}} f_{0} \\
& \frac{\partial f_{1}}{\partial t}+v_{0} \frac{\partial f_{1}}{\partial x}+\frac{e}{M} E \frac{\partial f_{i}}{\partial v}=\left\langle\sigma_{i e} v_{\mathrm{e}}\right\rangle n_{\mathrm{e}} f_{0} \\
& n_{i}=\int f_{i} d v, \quad j_{i}=\int v f_{i} d v
\end{aligned}
$$

where $f_{0}$ and $f_{i}$ are the distribution function of neutrals and ions, respectively. These functions satisfy boundary conditions:

$$
\begin{array}{r}
f_{0}(0, v, t)=\frac{1}{(2 \pi M T)^{3 / 2}} \exp \left(-\frac{M v^{2}}{2 T}\right) \\
\text { for positive velocities } \\
f_{i}(0, v, t)=0 \text { for negative velocities }
\end{array}
$$

In such a case $\left\langle\sigma_{i e} \nu_{\mathrm{e}}\right\rangle=\sigma_{\max } \nu_{\mathrm{e}}\left(T_{\mathrm{e}}\right) \exp \left(-U_{i} / T_{\mathrm{e}}\right)$, where $\sigma_{\max }$ - the maximal ionisation cross-section, $v_{\mathrm{e}}\left(T_{\mathrm{e}}\right)$, an average electron thermal velocity, $U_{i}$ the the ionisation potential.

For electrons, we can use a hydrodynamic model, as before:

$$
\begin{aligned}
& j_{d}=j_{i}+j_{\mathrm{e}} \\
& j_{\mathrm{e}}=\mu_{\perp}\left(e n_{\mathrm{e}} E-\frac{\partial E}{\partial x} \tau_{0} j_{d}\right) \\
& \frac{\partial E}{\partial x}=4 \pi e\left(n_{i}-n_{\mathrm{e}}\right)
\end{aligned}
$$

The computed results are shown in Fig. 6(a,b) in a comparison with those from a hydrodynamic model. One can easily see that taking into account span mode with single ionisation does not have a great influence on the resulting potential distribution (Fig. 6a). The electron density distribution (Fig. 6b) looks more consistent along the gap in a hybrid model in a comparison with that found from a hydrodynamic model. It is the result of an influence of neutrals and ionisation on the system processes, which were not taken into account in the hydrodynamic model. Nevertheless, the hydrodynamic model describes the system quite well and allows get exact analytical solutions.

\section{Conclusions}

For the first time, the original approach to use plasma accelerators with closed electron drifts and open walls, applicable for the creation of cost-effective low-maintenance plasma devices for production of ion beams converging towards the $z$-axis, was described. The pilot unit of such a plasma accelerator was created and tested. The first experimental results were obtained for a low-current and a high-current modes.

Based on the idea of continuity of current transfer in the considered system, exact analytical solutions, which describe an electric potential distribution along the acceleration gap, were found. It was shown

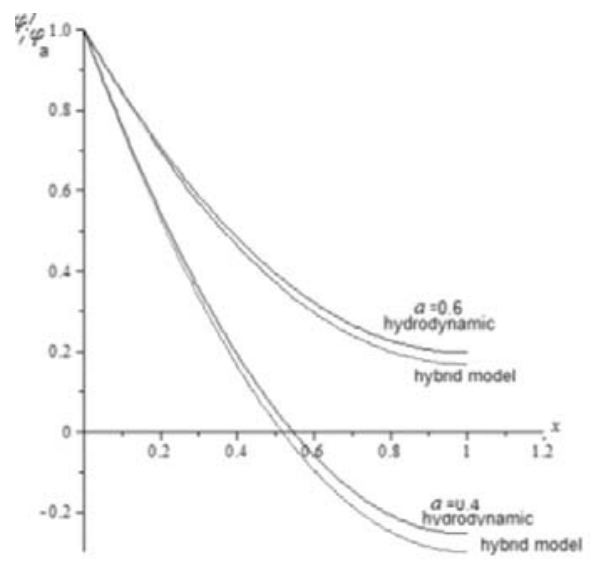

Fig. 6a. Potential distributions obtained from the hydrodynamic and hybrid models.

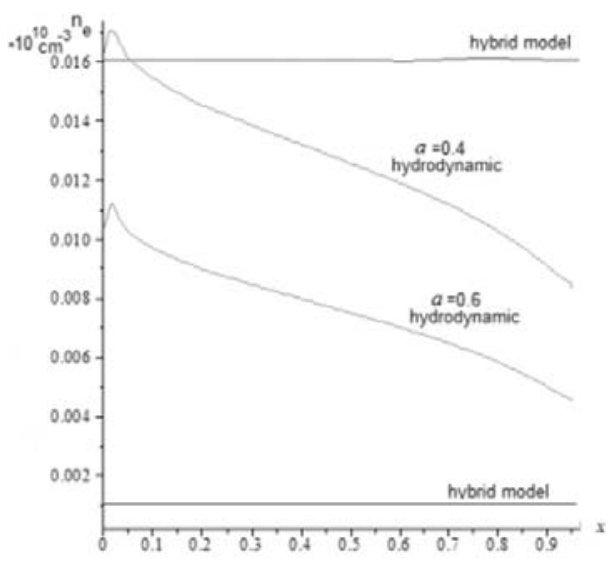

Fig. 6b. Electron density distributions obtained from the hydrodynamic and hybrid models. 
that the potential distribution is parabolic for different operation modes (i.e., in a low-current mode as well as in a high current quasi-neutral plasma mode) and it slightly depends on the electron temperature.

It was found that, under conditions at which all electrons were produced within the gap by impact ionisation only and propagated towards the anode (due to classical mobility in a transverse magnetic field), the full potential drop in the accelerating gap appears within the anode layer thickness. In a case when the gap length is smaller than the anode layer thickness, this potential drop is not completed. For the case when the gap length is larger than the anode layer, the considered potential drop exceeds the applied voltage.

For a high-current quasi-neutral mode, a general condition of a self-sustained discharge in the crossed $E \times H$ fields, taking into consideration both electron and ion dynamics peculiarity. The simulation results obtained from the hydrodynamic and hybrid model are in a good accordance, and their behaviour is similar to exact analytical solutions.

The presented plasma device is attractive for the creation of cost-effective and low-maintenance plasma lens with a positive space charge needed for focusing negative intense charge particles beams (electrons and negative ions). It creates opportunity to design low-cost and low-energy small rocket engines and can be useful for many other high-tech practical applications.
Acknowledgment. The work is partly supported by the grant of NAS of Ukraine no. 34-08-15 and no. PL-15-32.

This work was performed at the Institute for Nuclear Physics and Institute of Physics NASU in Kiev, Ukraine.

\section{References}

1. Goncharov, A. A., \& Brown, I. G. (2007). Plasma devices based on the plasma lens: A review of results and applications. IEEE Trans. Plasma Sci., 35(4), 986-991.

2. Goncharov, A., Dobrovolskiy, A., Dunets, S., Evsyukov, A., Litovko, I., Gushenets, V., \& Oks, E. (2011). Positive-space-charge lens for focusing and manipulating high-current beams of negatively charged particles. IEEE Trans. Plasma Sci., 39(6), 1408-1411.

3. Gushenets, V., Goncharov, A., Dobrovolskiy, A., Dunets, S., Litovko, I., Oks, E., \& Bugaev, A. (2013). Electrostatic plasma lens focusing of an intense electron beam in an electron source with a vacuum arc plasma cathode. IEEE Trans. Plasma Sci., 41(4), 2171-2174.

4. Morozov, A. I. (2008). Introduction to plasma dynamics. Moscow: Fismatlit.

5. Litovko, I., Goncharov, A., Dobrovolskiy, A., Najko, L., Najko, I., Gushenets, V., \& Oks, E. (2015). Computer modeling plasma optical devices (new results). Probl. Atom. Sci. Techn. (VANT), 95(1), 209-212.

6. Grishin, D., Leskov, L., \& Kozlov, N. (1983). Plasma accelerators. Moscow: Mashinostroenie. 